\title{
Identification of novel genes in aging osteoblasts using next- generation sequencing and bioinformatics
}

\author{
Yi-Jen Chen ${ }^{1,2}$, Wei-An Chang1,3, Ming-Shyan Huang ${ }^{4,5}$, Chia-Hsin Chen ${ }^{2,6}$, Kuan- \\ Yuan Wang ${ }^{7}$, Ya-Ling Hsu ${ }^{8}$ and Po-Lin Kuo ${ }^{1,8,9}$ \\ ${ }^{1}$ Graduate Institute of Clinical Medicine, College of Medicine, Kaohsiung Medical University, Kaohsiung, Taiwan \\ ${ }^{2}$ Department of Physical Medicine and Rehabilitation, Kaohsiung Medical University Hospital, Kaohsiung, Taiwan \\ ${ }^{3}$ Division of Pulmonary and Critical Care Medicine, Kaohsiung Medical University Hospital, Kaohsiung, Taiwan \\ ${ }^{4}$ Department of Internal Medicine, E-DA Cancer Hospital, Kaohsiung, Taiwan \\ ${ }^{5}$ School of Medicine, I-Shou University, Kaohsiung, Taiwan \\ ${ }^{6}$ Department of Physical Medicine and Rehabilitation, School of Medicine, Kaohsiung Medical University, Kaohsiung, Taiwan \\ ${ }^{7}$ Division of Geriatrics and Gerontology, Kaohsiung Medical University Hospital, Kaohsiung, Taiwan \\ ${ }^{8}$ Graduate Institute of Medicine, College of Medicine, Kaohsiung Medical University, Kaohsiung, Taiwan \\ ${ }^{9}$ Institute of Medical Science and Technology, National Sun Yat-Sen University, Kaohsiung, Taiwan \\ Correspondence to: Ya-Ling Hsu, email: hsuyl326@gmail.com \\ Po-Lin Kuo, email: kuopolin@seed.net.tw
}

Keywords: aging osteoblasts; next-generation sequencing; bioinformatics; microRNA; messenger RNA

Received: September 08, 2017 Accepted: October 27, $2017 \quad$ Published: November 28, 2017

Copyright: Chen et al. This is an open-access article distributed under the terms of the Creative Commons Attribution License 3.0 (CC BY 3.0), which permits unrestricted use, distribution, and reproduction in any medium, provided the original author and source are credited.

\section{ABSTRACT}

During the aging process, impaired osteoblastic function is one key factor of imbalanced bone formation and age-related bone loss. The aim of this study is to explore the differentially expressed genes in normal and aged osteoblasts and to identify genes potentially involved in age-related alteration in bone physiology. Based on next generation sequencing and bioinformatics analysis, 12 differentially expressed microRNAs and 22 differentially expressed genes were identified. Up-regulation of miR204-5p was validated in an array of osteoporotic hip fracture in the Gene Expression Omnibus database (GSE74209). The putative targets for miR-204-5p were Kruppellike factor 7 (KLF7) and SRY-box 11 (SOX11). Ingenuity Pathway Analysis identified SOX11, involved in osteoarthritis pathway and differentiation of osteoblasts, together with miR-204-5p, a potential upstream regulator, suggesting the critical role of miR-204$5 p-S O X 11$ regulation in the aging process of human bones. In addition, as semaphorin 3A (SEMA3A) and ephrin type-A receptor 5 (EPHA5) were involved in nervous system related biological functions, we postulated a potential linkage between SEMA3A, EPHA5 and development of neurogenic heterotopic ossification. Our findings implicate new candidate genes in the diagnosis of geriatric musculoskeletal disorders, and provide novel insights that may contribute to the elaboration of new biomarkers for neurogenic heterotopic ossification.

\section{INTRODUCTION}

Life expectancy has increased during the past decades, and issues related to global aging are growing. At present, the average percentage of elderly around the world is about $15 \%$, and this number is expected to increase to approximately $20 \%$ by 2050 [1]. With aging, body systems and organs undergo physiological changes, resulting in increased comorbidities [2]. 
Bone structure consists of trabecular bone and cortical bone. Trabecular bone is more metabolically active and its bone mineral density begins to decrease in young adulthood. Cortical bone provides mainly structural support, and its bone mineral density remains stable until middle age and then begins to decrease [3]. Postmenopausal increase in bone turnover and bone porosity is the primary factor for women having more overall cortical bone loss than men throughout life [4]. The maintenance of bone health relies on the balanced remodeling of osteoblasts and osteoclasts. Osteoblasts are responsible for bone formation, whereas osteoclasts are responsible for bone resorption [5]. Disequilibrium of osteoblast and osteoclast activities related to aging, metabolic diseases and hormonal changes may lead to bone fragility and clinical disorders such as osteoporosis [6].

MicroRNAs are small non-coding RNAs of 20-22 nucleotides which regulate gene expressions through a post-transcriptional manner, acting as key regulators in various biological processes [7]. Novel targets in many biological functions and human diseases have been discovered by microRNA profiling, including bone remodeling $[8,9]$ and osteoarthritis (OA) [10, 11]. Dysregulated microRNAs were also identified as affecting the differentiation and proliferation of multipotent mesenchymal stem cells (MSCs), thereby regulating bone formation [12-14]. In the era of an aging population, research on the aging processes of different organ systems using microarray and next generation sequencing (NGS) approaches has also evolved [1517]. NGS technique provides high-throughput genomic profiling of the whole genome, including DNA, RNA and small RNA sequencing, and DNA methylation [18]. The differentially expressed profiling results obtained from NGS require further analysis using bioinformatics approaches to identify candidate genes and/or microRNAs of interest. In this study, we sought to identify potential microRNA-mRNA interactions in aged osteoblasts using different bioinformatics databases and tools, including Ingenuity ${ }^{\circledR}$ Pathway Analysis (IPA), Database for Annotation, Visualization and Integrated Discovery (DAVID) [19], Gene Expression Omnibus (GEO) [20], and miRmap [21].

Both OA and osteoporosis are common age-related musculoskeletal disorders. Currently, the diagnosis of $\mathrm{OA}$ and osteoporosis relies largely on imaging studies, and disease severity does not necessarily correlate with clinical symptoms. The aim of this study is to explore the differentially expressed genes in aged osteoblasts and to identify potential genes involved in age-related alterations in bone physiology. Through the NGS and bioinformatics approaches, we hope to provide novel perspectives in the future development of biomarkers in advancing diagnosis and evaluation of therapeutic efficacy of age-related bone diseases among the geriatric population.

\section{RESULTS}

\section{Confirmation of cell senescence of osteoblasts at late passages}

The cellular aging of human osteoblasts, like many other cell types, demonstrated characteristic morphological changes and increased proportion of cells with positive $\beta$-galactosidase staining [22]. We observed the serial morphological changes of human osteoblasts of different passages. As the osteoblasts were cultured to later passages, the cell morphologies changed from thin, spindle shape to flattened, irregular shape with increased intracellular debris (Figure 1A). The proportion of $\beta$-galactosidase positive cells also increased in the later passages, as shown in Figure 1B, indicating the osteoblast senescence.

\section{Identification of potential microRNA-mRNA interactions in aging osteoblasts}

Studies have indicated the importance of microRNA regulation on the gene expression in the aging process of human musculoskeletal system [23-25]. To study the microRNAs potentially involved in the aging process of osteoblasts, the NGS results of small RNA-seq from human primary osteoblasts of first (P1) and eighth (P8) passages were obtained. MicroRNAs with $>2.0$-fold differential expression and reads per million $(\mathrm{RPM})>10$ were selected. Twenty-nine microRNAs were identified, as listed in Table 1 . To identify potential microRNAmRNA interactions in aging osteoblasts, we first analyzed the putative targets of 10 up-regulated microRNAs and 19 down-regulated microRNAs from NGS results using miRmap microRNA putative target predictor, and selected targets with miRmap scores $>99.0$ that indicated high predictive strength of repression. A total of 381 target genes (412 potential interactions) predicted by 10 upregulated microRNAs, and 606 genes (790 potential interactions) predicted by 19 down-regulated microRNAs were identified. We then matched the selected target genes to our RNA-seq with $>2.0$ fold down- or up-regulated mRNAs, using the "Compare Dataset" tool in the IPA. Matched results identified 22 target genes (11 downregulated genes and 11 up-regulated genes), as shown in Figure 2A. The heatmap analysis of 29 differentially expressed microRNAs with z-score values was shown in Figure 2B.

\section{Differentially expressed miR-204-5p and miR- $335-3 p$ in osteoporotic hip bone}

The 5 up-regulated microRNAs and 7 downregulated microRNAs with corresponding putative targets of aged osteoblasts identified via Venn diagram analysis (11 down-regulated genes and 11 up-regulated genes, 
respectively) are listed in Table 2. To confirm whether these identified microRNAs are of clinical significance in the aging process of bones, we searched the GEO database for related arrays in age-related bone diseases such as OA and osteoporosis, and found a related array (GEO accession: GSE74209). The array contained bone specimens from six women with osteoporotic femoral neck fractures, and six women with hip OA but no osteoporosis [26]. The 12 differentially expressed microRNAs were individually analyzed with corresponding identifiers from the ID column of the array dataset for expression values. The results showed that miR-204-5p was significantly up-regulated, whereas miR-335-3p was significantly down-regulated, in women with osteoporotic hip fractures compared to those with hip OA but no osteoporosis (Figure $3 \mathrm{~A}$ and $3 \mathrm{~B}$ ). As listed in Table 2, KLF7 and SOX11 were the predicted targets of miR-204-5p, while KIAA1462 and SVIP were targets of miR-335-3p.

We further sought to identify diseases and functions associated with the 22 target genes analyzed using IPA software. Three networks associated with cancer, organ injury and abnormality, tissue morphology, endocrine system disorders, cellular development, cell-mediated immune response and cellular movement were identified, with networks 1 and 2 having higher scores and 10 focused molecules (Table 3). Matched to the putative targets analyzed in the previous section, three of the four predicted targets (KLF7, SOX11 and SVIP) were involved in network 2 (Figure 4A). Of the two microRNAs identified, miR-204-5p was one of the upstream regulators ( $p$-value of overlap $=8.15 \mathrm{E}-03$, targeting EPHA5, PID1, $P L A G 1$ and SOX11 in this dataset) identified in the IPA analysis result (Figure 4B). Using the overlay tool, we identified the molecules involved in the osteoarthritis pathway, Wnt/ $\beta$-catenin pathway, and differentiation of osteoblasts (marked in purple in Figure 4B), with SOX11 the only molecule simultaneously involved. We then analyzed the binding site and sequence of miR-204-5p in the 3'UTR of SOX11 in miRmap, TargetScan and miRDB. Three target sites of miR-204-5p on SOX11 3'UTR were identified in all of the above microRNA target prediction databases, including the position of 589-595 (miRmap score 99.41), 985-991 (miRmap score 96.12) and 59105916 (miRmap score 93.40) (Figure 5). The results suggest that the regulation of miR-204-5p on SOX11 may play a critical role in the aging process of human bone.

\section{Analysis of potential molecules involved in miR- 204-5p regulation on $S O X 11$}

To determine the potential interactions between miR204-5p-SOX11 regulation and other downstream effectors
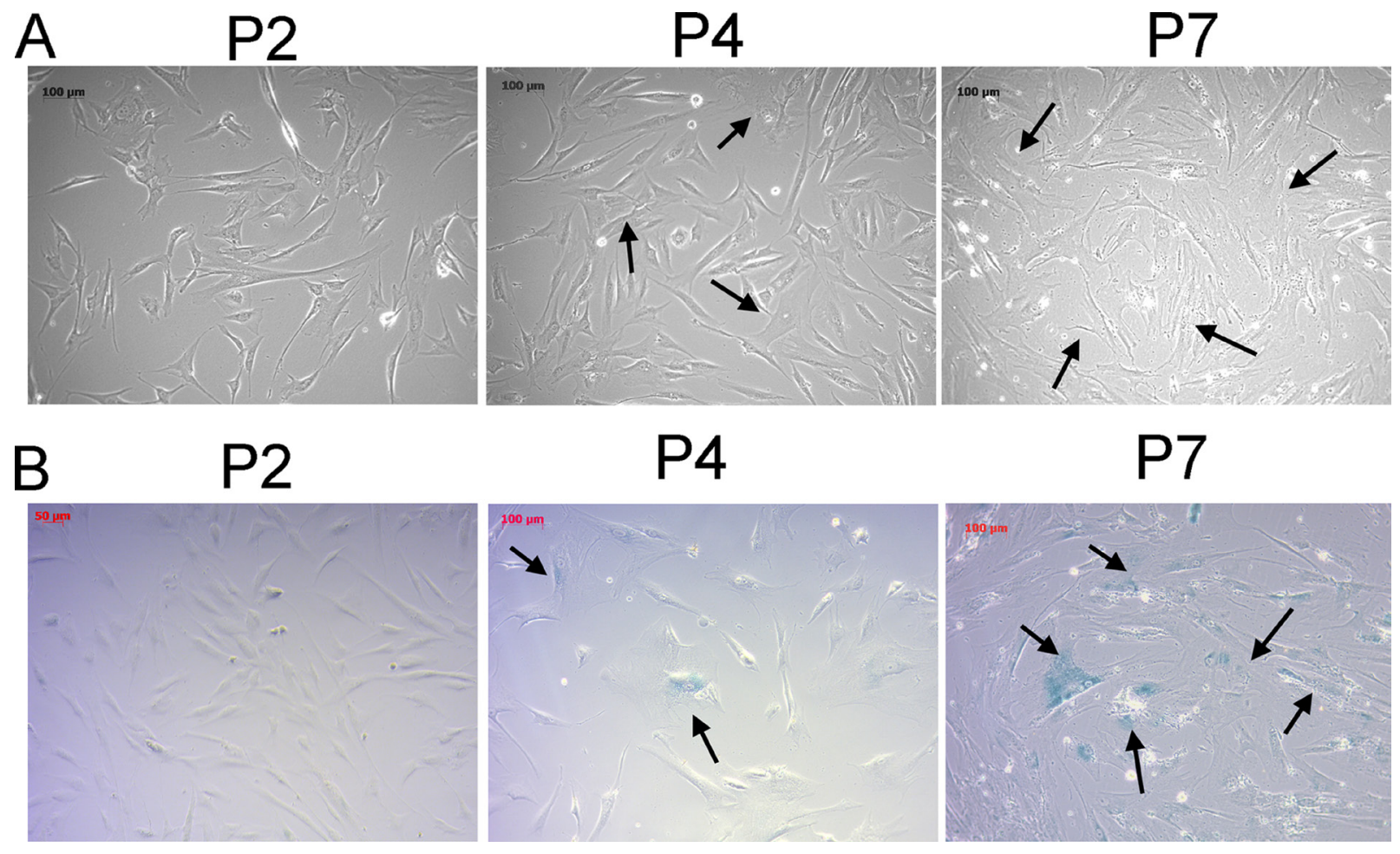

Figure 1: Morphological changes and $\beta$-galactosidase staining of human primary osteoblasts of different passages confirmed the osteoblast senescence at later passages. The morphological changes (A) and $\beta$-galactosidase staining (B) of human primary osteoblasts of second, fourth, and seventh passages were identified. The morphologies of young osteoblasts were thin and spindleshaped. Increased number of cells with flattened and irregularly-shaped morphologies and increased intracellular debris were observed at later passages (arrow). The cells with blue staining indicated $\beta$-galactosidase-positive cells (arrow), representing senescent cells. The cells were observed under light microscope at 10X field. 
Table 1: Differentially expressed microRNAs identified from P1 and P8 osteoblasts

\begin{tabular}{|c|c|c|c|c|c|}
\hline miRNA name & precursor & $\begin{array}{c}\text { OB-P8 seq } \\
\text { (norm) }\end{array}$ & $\begin{array}{c}\text { OB-P1 seq } \\
\text { (norm) }\end{array}$ & $\begin{array}{l}\text { Fold Change } \\
\text { OB-P8/OB-P1 }\end{array}$ & Direction of Change \\
\hline hsa-miR-376a-5p & hsa-mir-376a-1 & 94.85 & 19.73 & 4.81 & Up \\
\hline hsa-miR-204-5p & hsa-mir-204 & 294.77 & 64.6 & 4.56 & $\mathrm{Up}$ \\
\hline hsa-miR-136-5p & hsa-mir-136 & 143.84 & 41.23 & 3.49 & Up \\
\hline hsa-miR-137 & hsa-mir-137 & 37.94 & 12.13 & 3.13 & $\mathrm{Up}$ \\
\hline hsa-miR-132-3p & hsa-mir-132 & 207.12 & 67.07 & 3.09 & Up \\
\hline hsa-miR-181c-5p & hsa-mir-181c & 224.29 & 77.33 & 2.90 & Up \\
\hline hsa-miR-193a-3p & hsa-mir-193a & 122.83 & 43.4 & 2.83 & Up \\
\hline hsa-miR-4792 & hsa-mir-4792 & 57.39 & 23.87 & 2.40 & Up \\
\hline hsa-miR-497-5p & hsa-mir-497 & 72.28 & 33.14 & 2.18 & Up \\
\hline hsa-miR-27a-3p & hsa-mir-27a & 5256.87 & 2456.33 & 2.14 & $\mathrm{Up}$ \\
\hline hsa-miR-424-5p & hsa-mir-424 & 71.56 & 151.4 & -2.12 & Down \\
\hline hsa-miR-708-5p & hsa-mir-708 & 15.37 & 32.84 & -2.14 & Down \\
\hline hsa-miR-20a-5p & hsa-mir-20a & 106.5 & 227.74 & -2.14 & Down \\
\hline hsa-miR-708-3p & hsa-mir-708 & 16.09 & 34.82 & -2.16 & Down \\
\hline hsa-miR-22-5p & hsa-mir-22 & 21.61 & 46.85 & -2.17 & Down \\
\hline hsa-miR-222-5p & hsa-mir-222 & 25.45 & 55.92 & -2.20 & Down \\
\hline hsa-miR-1180-3p & hsa-mir-1180 & 30.98 & 68.94 & -2.23 & Down \\
\hline hsa-miR-148a-5p & hsa-mir-148a & 48.99 & 109.09 & -2.23 & Down \\
\hline hsa-miR-335-5p & hsa-mir-335 & 589.9 & 1331.53 & -2.26 & Down \\
\hline hsa-miR-138-1-3p & hsa-mir-138-1 & 12.37 & 31.56 & -2.55 & Down \\
\hline hsa-miR-1260b & hsa-mir-1260b & 359.61 & 932.17 & -2.59 & Down \\
\hline hsa-miR-1260a & hsa-mir-1260a & 321.06 & 841.03 & -2.62 & Down \\
\hline hsa-miR-424-3p & hsa-mir-424 & 39.98 & 120.63 & -3.02 & Down \\
\hline hsa-miR-452-5p & hsa-mir-452 & 38.78 & 122.5 & -3.16 & Down \\
\hline hsa-miR-224-5p & hsa-mir-224 & 113.59 & 373.32 & -3.29 & Down \\
\hline hsa-miR-378a-3p & hsa-mir-378a & 121.39 & 444.83 & -3.66 & Down \\
\hline hsa-miR-335-3p & hsa-mir-335 & 791.86 & 3652.64 & -4.61 & Down \\
\hline hsa-miR-146b-3p & hsa-mir-146b & 11.29 & 69.04 & -6.12 & Down \\
\hline hsa-miR-146b-5p & hsa-mir-146b & 186.47 & 1794.21 & -9.62 & Down \\
\hline
\end{tabular}

in our aging osteoblast database, we identified miR-204-5p as an upstream regulator in IPA results for all differentially expressed genes. The network of miR-204-5p related molecules with overlay tool in the IPA was used, and revealed that $A L P L, C Y P 1 B 1, E G R 1$, GREM1, IGFBP5, $P R D M 1$ and $S O X 11$ are associated with differentiation of osteoblast and muscle cells, bone mineralization and mineral density, and osteoclastogenesis (Table 4).

\section{Identification of differentially expressed genes in aged human osteoblasts and the potential involved pathways}

To further identify pathways involved in the aging process of human bones, we performed RNA-seq by NGS to compare the differentially expressed genes between P1 and P8 human osteoblasts. Differentially expressed genes between P1 and P8 osteoblasts with at least two-fold upregulation or down-regulation were selected from the NGS data. The NGS results identified 504 up-regulated genes and 385 down-regulated genes in P8, compared to P1 osteoblasts. To determine the potential pathways involved in these differentially expressed genes, the list of these differentially expressed genes was uploaded to IPA for further analysis. IPA analysis revealed 133 significant canonical pathways that were associated with differentially expressed genes of our RNA-seq database. Among the top 10 canonical pathways identified, 30 molecules were involved in $\mathrm{OA}$ and rheumatoid arthritis (RA) related pathways (16 up-regulated genes 
and 14 down-regulated genes in our database). The differentially expressed genes were categorized into 25 networks, where diseases and functions annotation such as bone mineral density, differentiation of osteoblasts, osteoarthritis and damage of cartilage tissue were selected to identify related genes. Network analysis is shown in Figure 6, where $S O X 11$ was interconnected between the networks of osteoblast differentiation and bone mineral density, and was predicted to inhibit EGR1 and activate $P R D M 1$, two molecules in the bone mineral density network.

\section{Upregulation of EPHA5 and $S E M A 3 A$ involved in the axon guidance pathway}

The 22 genes with potential microRNA-mRNA interactions were input into the DAVID database to analyze the related biological processes and pathways, and address potential mechanisms involved in aging osteoblasts. The KEGG pathway analysis in DAVID database was used, and set the cutoff value at $\mathrm{EASE}=1$ to avoid falsenegative results in a smaller gene list containing only 22 genes. Result indicated 2 genes, EPHA5 and SEMA3A, were involved in the axon guidance pathway (Table 5). The results were identical to IPA network analysis, where overlay canonical pathway of axon guidance signaling in network 1 from network analysis of these 22 candidate genes revealed the involvement of EPHA5 and SEMA3A (Figure 7). As determined by gene ontology results from functional annotation analysis in DAVID database, the terms of biological processes of the genes of interest are shown in Figure 8. Most of the above-mentioned genes (KLF7, SOX11, EPHA5, and SEMA3A) were involved in nervous system related biological functions, including axon guidance, sympathetic nervous system development and dendrite morphogenesis.

\section{DISCUSSION}

Bone structure consists of trabecular bone and cortical bone. Osteoblasts are distributed in the inner layer of the periosteum and along the trabeculae. They are derived from MSCs, and differentiate into osteocytes [27]. During the aging process, impaired osteoblastic function is the consequence of decreased proliferation and differentiation, and increased cellular senescence of
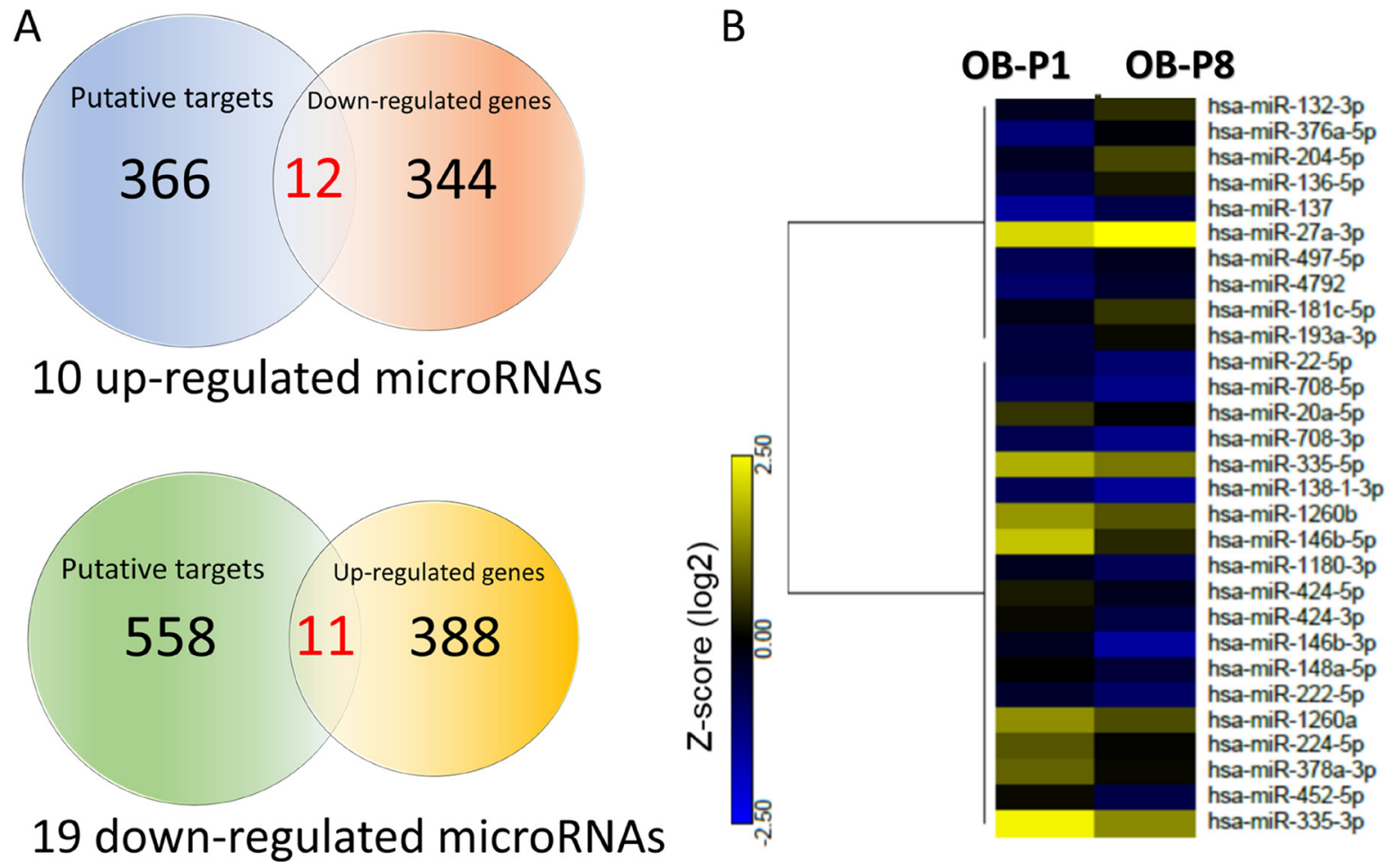

Figure 2: Identification of differentially expressed microRNAs and genes with potential microRNA-mRNA interactions in human primary osteoblasts. (A) Next generation sequencing (NGS) analysis identified 504 up-regulated genes and 385 downregulated genes in human primary osteoblasts of eighth passage (P8), compared to first passage (P1) (> 2.0-fold change), where 399 upregulated genes and 355 down-regulated genes were mapped using Ingenuity Pathway Analysis (IPA) database. In addition, 10 up-regulated microRNAs and 19 down-regulated microRNAs were selected $(>2.0$-fold change and threshold of reads per million $(\mathrm{RPM})>10)$, which predicted 381 and 606 putative targets by miRmap (score $\geq 99.0$ ), respectively. Among all identified putative targets, 378 targets predicted by 10 up-regulated microRNAs and 599 targets predicted by 19 down-regulated microRNAs were mapped in IPA database. These selected targets and differentially expressed genes were matched by the "Compare Dataset" tool in the IPA, and revealed 22 potential microRNAmRNA interactions. "One of the mapped genes was not identified in our NGS dataset. (B) The heatmap analysis of differentially expressed microRNAs from P1 and P8 osteoblasts with z-score values were shown. 
Table 2: Genes selected between putative targets of microRNA and differentially expressed genes from NGS database

\begin{tabular}{|c|c|c|c|}
\hline Up-regulated microRNA & Target down-regulated mRNA & Gene name & Fold-change (P8 vs. P1) \\
\hline \multirow[t]{6}{*}{ hsa-miR-497-5p } & ADAMTS12 & $\begin{array}{l}\text { ADAM metallopeptidase with } \\
\text { thrombospondin type } 1 \text { motif, } 12\end{array}$ & 5.31 \\
\hline & $C D 40$ & CD40 molecule & 4.28 \\
\hline & COL12A1 & collagen, type XII, alpha 1 & 3.89 \\
\hline & SIK1 & salt-inducible kinase 1 & 3.38 \\
\hline & PID1 & $\begin{array}{l}\text { phosphotyrosine interaction domain } \\
\text { containing } 1\end{array}$ & 3.22 \\
\hline & $R A B 3 I P$ & RAB3A interacting protein & 5.88 \\
\hline \multirow[t]{2}{*}{ hsa-miR-193a-3p } & $A J U B A$ & ajuba LIM protein & 3.38 \\
\hline & NT5DC3 & 5'-nucleotidase domain containing 3 & 2.85 \\
\hline \multirow[t]{2}{*}{ hsa-miR-204-5p } & $K L F 7$ & Kruppel-like factor 7 & 2.02 \\
\hline & SOX11 & SRY (sex determining region Y)-box 11 & 6.97 \\
\hline hsa-miR-181c-5p & $R A B 3 I P$ & RAB3A interacting protein & 5.88 \\
\hline hsa-miR-27a-3p & SLC7A11 & solute carrier family 7 member 11 & 2.55 \\
\hline $\begin{array}{l}\text { Down-regulated } \\
\text { microRNA }\end{array}$ & Target up-regulated mRNA & Gene name & Fold-change (P8 vs. P1) \\
\hline hsa-miR-1260a & $C A C N B 4$ & $\begin{array}{l}\text { calcium channel, voltage-dependent, beta } \\
4 \text { subunit }\end{array}$ & 9.51 \\
\hline hsa-miR-1260b & $C A C N B 4$ & $\begin{array}{l}\text { calcium channel, voltage-dependent, beta } \\
4 \text { subunit }\end{array}$ & 9.51 \\
\hline \multirow[t]{3}{*}{ hsa-miR-20a-5p } & $C E L F 2$ & CUGBP Elav-like family member 2 & 5.90 \\
\hline & EPHA5 & ephrin type-A receptor 5 & 20.28 \\
\hline & FGD4 & $\begin{array}{l}\text { FYVE, RhoGEF and PH domain } \\
\text { containing } 4\end{array}$ & 2.50 \\
\hline \multirow[t]{4}{*}{ hsa-miR-424-5p } & $E 2 F 7$ & E2F transcription factor 7 & 2.10 \\
\hline & PDK4 & pyruvate dehydrogenase kinase, isozyme 4 & 8.54 \\
\hline & PLAG1 & pleiomorphic adenoma gene 1 & 2.65 \\
\hline & SEMA3A & semaphorin $3 \mathrm{~A}$ & 9.58 \\
\hline \multirow[t]{2}{*}{ hsa-miR-335-3p } & KIAA1462 & KIAA1462 & 2.12 \\
\hline & SVIP & small VCP interacting protein & 2.74 \\
\hline hsa-miR-335-5p & KIAA1462 & KIAA1462 & 2.12 \\
\hline hsa-miR-224-5p & $M O X D 1$ & monooxygenase DBH-like 1 & 3.28 \\
\hline
\end{tabular}

MSCs, leading to imbalanced bone formation and agerelated bone loss $[3,28]$.

Our current study, based on NGS and bioinformatic analysis, identified 22 differentially expressed genes and 12 differentially expressed microRNAs potentially involved in aging osteoblasts. We further analyzed the 12 microRNAs in the GEO database of osteoporotic fracture array data (GSE74209), and found miR-204-5p to be up-regulated in osteoporotic fracture bone, which was also a potential upstream regulator of the 22 candidate genes in aging osteoblasts identified in IPA analysis. The putative target genes for miR-204-5p in our database were identified as KLF7 and SOX11, with SOX11 the gene of interest. Here, we proposed a novel finding of miR-204-5p-SOX11 regulation during the process of geriatric musculoskeletal physiological changes.
MiR-204-5p has been reported to be a tumor suppressor microRNA in different cancer types [29-31]. Huang et al. demonstrated that miR-204 inhibited osteogenesis and promoted adipogenesis of bone marrow stem cells through negative regulation of Runx2, an important transcription factor for chondrogenesis and osteogenesis [32]. In a rat model of osteoporosis, miR204-5p was one of the microRNAs associated with bone metabolism in bone marrow osteoblastic cells [33]. In our current study, we found that miR-204-5p was 4.56-fold up-regulated in aged osteoblasts, which was validated in a GEO array of trabecular bone specimens from osteoporotic hip fractures in elderly women [26]. The results indicate that miR-204-5p may be a key regulator of altered bone health in geriatric population. 
$S O X 11$ is one of the $S O X C$ transcription factors comprised of $S O X 4, S O X 11$ and $S O X 12$. SOXC proteins are expressed in various types of progenitor cells, and potentiate cell survival and differentiation related pathways, regulating prenatal structural development $[34,35]$. Deletion of Sox 11 in mice results in reduced ossification and skeletal malformations in multiple sites, suggesting the regulatory role of SOX11 in enhancing osteoblast differentiation [36, 37]. In a mouse OA model performed by Kan et al., the expression of Sox 11 in the degraded cartilage of the medial knee joint was significantly decreased 4 weeks after OA induction, compared to the lateral side or sham-operated knee joint [38]. Xu et al. also proved promotion of ectopic bone formation in Sox11-overexpressed MSCs in vivo, and fracture healing in rats [39]. To date, only a few studies
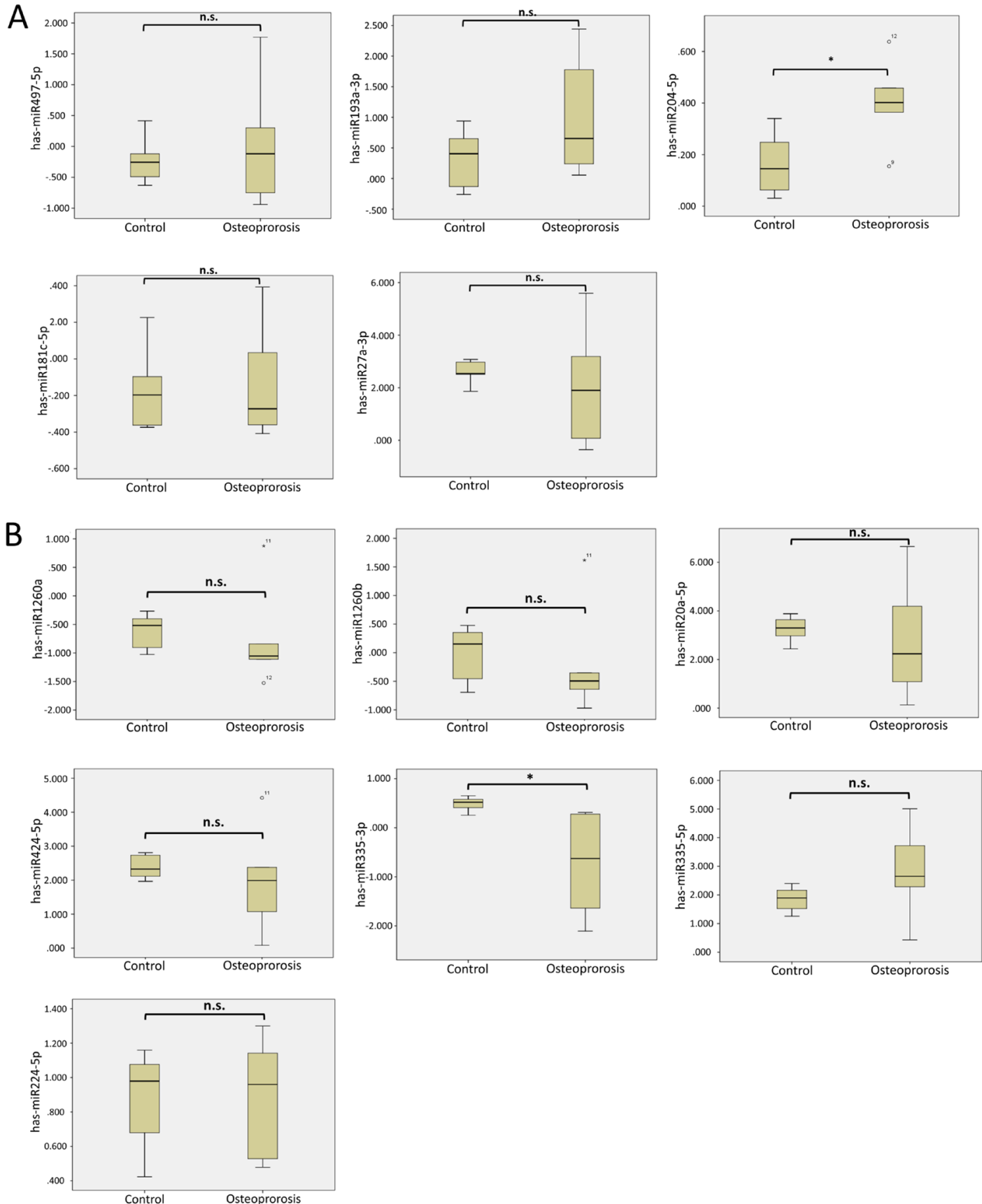

Figure 3: Analysis of 12 microRNAs with potential interactions in the GEO database. The 5 up-regulated microRNAs (A) and 7 down-regulated microRNAs (B) were validated in a related array (GSE74209) from the GEO database. Significant upregulation of miR-204-5p and downregulation of miR-335-3p were observed in women with osteoporotic hip fractures, when compared to those with hip osteoarthritis without osteoporosis. ${ }^{*}$ indicated $p<0.05$, and n.s. indicated no statistical significance. (ID reference: miR-497-5p, 2829; miR-193a-3p, 2567; miR-204-5p, 2564; miR-181c-5p, 861; miR-27a-3p, 845; miR-1260a, 411; miR-1260b, 382; miR-20a-5p, 4278; miR424-5p, 587; miR-335-3p, 321; miR-335-5p, 1996; miR-224-5p, 2367). 
Table 3: Networks associated with genes targeted by miRNAs differentially expressed in osteoblasts

\begin{tabular}{|c|c|c|c|c|}
\hline & Top diseases and functions & Score & Focus molecules & Molecules in network \\
\hline 1 & $\begin{array}{l}\text { Cancer, organismal injury } \\
\text { and abnormalities, tissue } \\
\text { morphology }\end{array}$ & 24 & 10 & $\begin{array}{l}\text { 2' 5' oas, Act1, ADAMTS12, AJUBA, Akt, } \\
\text { CD40, CD80/CD86, CELF2, Chil3/Chil4, } \\
\text { Cr3, EFNA1, EPHA5, ERK, ERK1/2, F } \\
\text { Actin, Fascin, Fcer, FGD4, GTPase, Hla-abc, } \\
\text { IGHE, IL6, Jnk, N-Cadherin, Pde4, PDK4, } \\
\text { PID1, Proinsulin, RAB3IP, RET, SEMA3A, } \\
\text { SEMA3D, sphingomyelinase, TRAF, Traj18 }\end{array}$ \\
\hline 2 & $\begin{array}{l}\text { Cancer, endocrine system } \\
\text { disorders, organismal injury } \\
\text { and abnormalities }\end{array}$ & 24 & 10 & $\begin{array}{l}\text { Adaptor protein 2, ADSS, ATOH7, BSN, } \\
\text { CACNB1, CACNB4, CCND1, CGB3, } \\
\text { COL12A1, DCN, E2F7, ELAVL1, Hspg, } \\
\text { IER2, IGIP, KLF7, SIK1, miR-16-5p, miR- } \\
\text { 3175, miR-486-3p, MTORC1, N-Cadherin, } \\
\text { NT5DC3, PLAG1, RARA, RET, SLC7A11, } \\
\text { SNRK, SOX11, SVIP, TGFB1, TMSB15A, } \\
\text { VEGFA, VPREB3, WNT3A }\end{array}$ \\
\hline 3 & $\begin{array}{l}\text { Cellular development, cell- } \\
\text { mediated Immune response, } \\
\text { cellular movement }\end{array}$ & 4 & 2 & $\begin{array}{l}\text { GLRX3, KIAA1462, miR-1249-5p, miR-149- } \\
\text { 3p, miR-1972, miR-2110, miR-3103-5p, miR- } \\
\text { 4271, miR-5584-5p, miR-6967-5p, MOXD1, } \\
\text { SUMO3, TMEM25 }\end{array}$ \\
\hline
\end{tabular}

have reported the dysregulation of SOX11 in human musculoskeletal diseases. It was proposed that deletion or point mutation of SOX11 may be associated with CoffinSiris syndrome, a congenital neurodevelopmental disorder, which results in cranial and skeletal malformations [40]. Kan et al. investigated 10 human cartilage samples obtained during total knee arthroplasty, which revealed graded decreases in SOX11 protein expression in more severely degraded human articular cartilages [38]. In our study, we proposed the role of miR-204-5pSOX11 regulation in aging osteoblasts, and the potential downstream regulation of BMPR1A/Runx2 signaling by $S O X 11$ (Figure 4B), a signaling pathway involved in osteoporosis and OA [41, 42]. Evidence suggests that SOX11 not only serves as a key transcriptional factor in embryonic development of the skeletal system, but also take part in maintaining joint homeostasis and the bone healing process. Together, the evidence provides novel insights into investigating the physiological and pathological geriatric changes of the musculoskeletal system.

Using IPA analysis, we identified 10 out of 22 candidate genes from aged osteoblasts to be grouped into one network, where cyclin D1 (CCND1) served as a connecting hub (Figure 4A). CCND1 regulates transition of cell cycle from G1 phase to $\mathrm{S}$ phase and tumorigenesis through increased cell proliferation [43]. Cellular senescence has been proposed to be involved in the pathogenesis of OA and age-related bone loss [44]. MiR-204-5p overexpression has been found to induce downregulation of $C C N D 1$, resulting in differentiation of human MSCs toward adipogenic rather than osteogenic lineage [45]. Zhu et al. also found grade-dependent decrease in mRNA and protein expressions of CCND1 among the knee OA population, compared to healthy controls [46]. These findings supported our results regarding miR-204-5p regulation in aging bone process, where up-regulated miR-204-5p was postulated to downregulate SOX11 and be involved in the cell cycle arrest of osteoblasts, leading to clinical observations of increased bone loss among the geriatric population.

The bioinformatics approach in our study suggests that differentially expressed SEMA3A and EPHA5 in long-term passaged osteoblasts may be associated with biological functions of axon guidance, sympathetic nervous system development and dendrite morphogenesis. This information provides insight into the potential clinical relevance and connection between aging bone and molecular mechanisms of neurogenic heterotopic ossification (HO), a feature of mature bone formation in soft tissue after central nervous system (CNS) injuries like brain and spinal cord $[47,48]$. The etiology of HO still lacks consensus, with bone morphogenetic protein $(B M P)$ being the most widely proposed molecule involved in the mechanism of HO. Salisbury and colleagues proposed the contribution of neuroinflammation to the migration of osteogenic cells from endoneurial progenitors, and further $\mathrm{HO}$ formation in mouse quadriceps muscle [49, 50]. In addition, neuroinflammation promotes increased permeability of blood-nerve-barrier, which facilitates progenitor cell penetration [51]. These findings suggest evidence of interconnection between bone formation and sensory innervation, and may provide a clinical explanation of $\mathrm{HO}$ formation in traumatic CNS injuries.

EPHA5 belongs to the receptor tyrosine kinase family. Studies have suggested EPHA5 as a candidate 

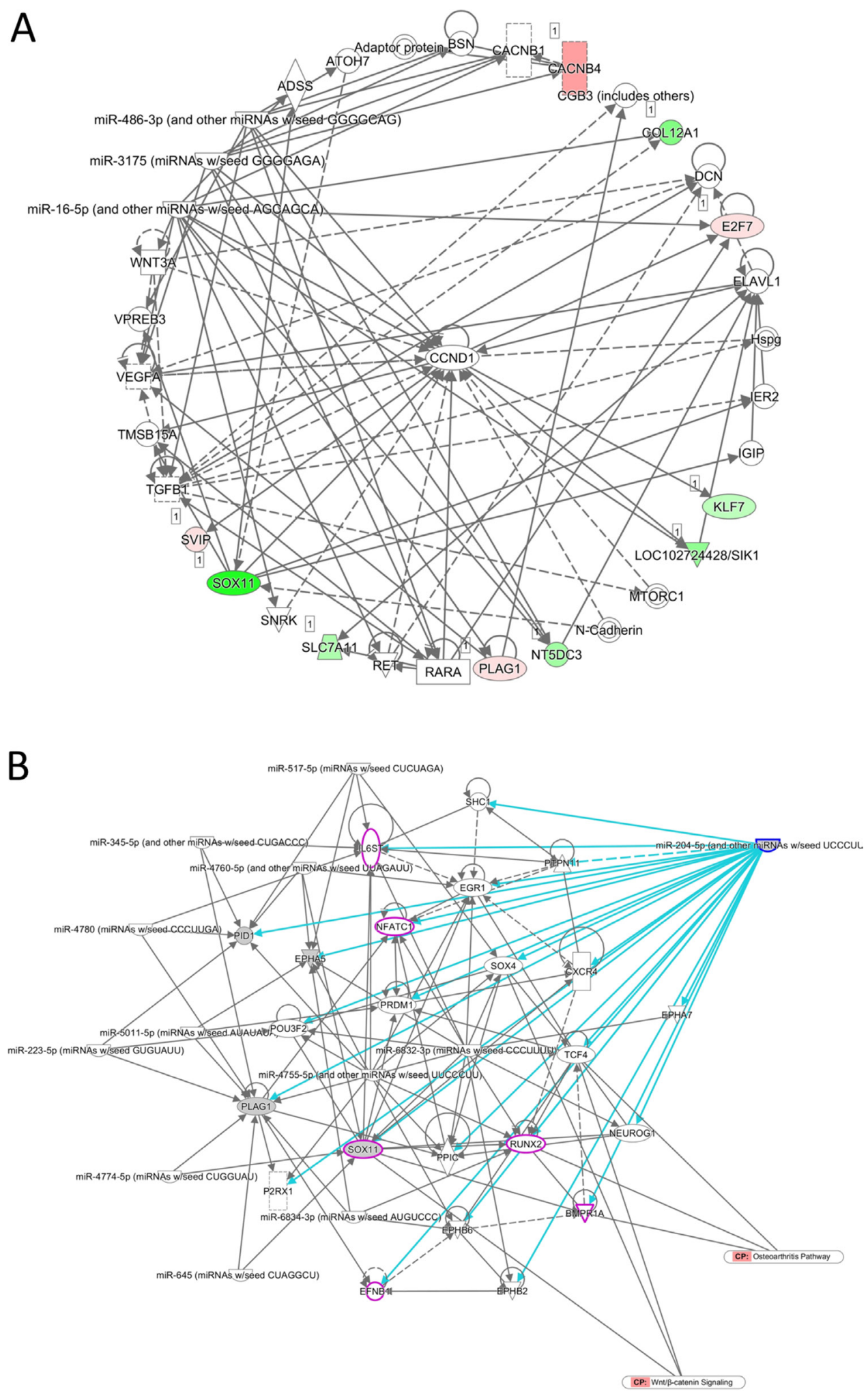

Figure 4: Prediction of network of genes involved in the regulation of miR-204-5p in aging human osteoblasts. (A) The 22 candidate genes identified from human osteoblasts were analyzed by IPA for network analysis. The network analysis revealed 10 out of 22 genes involved in a network associated with cancer, endocrine system disorders, organismal injury and abnormalities, and three of the four putative targets (KLF7, SOX11 and SVIP) by miR-204-5p and miR-335-3p predictions were involved. Genes colored in green indicated down-regulated expressions, and genes in red indicated up-regulated expressions in our dataset. (B) miR-204-5p was analyzed by IPA as a potential upstream regulator in our dataset. Using the overlay tool in IPA, molecules involved in the canonical osteoarthritis pathway, Wnt/ $\beta$-catenin pathway, and differentiation of osteoblasts were marked in purple, where SOX11 was the molecule simultaneously involved. 
Table 4: Potential molecules involved in miR-204-5p regulation on SOX11

\begin{tabular}{lll}
\hline Analysis & Function & Molecules \\
\hline miR-204-5p network & Bone mineral density & ALPL, EGR1, PRDM1 \\
& Differentiation of muscle cells & EGR1, IGFBP5, SOX11 \\
& Differentiation of osteoblast & ALPL, GREM1, IGFBP5, SOX11 \\
& Mineralization of bone & ALPL, GREM1, SOX11 \\
& Osteoclastogenesis & CYP1B1, PRDM1 \\
\hline
\end{tabular}

Table 5: KEGG pathway analysis of 22 candidate genes with potential microRNA-mRNA interactions

\begin{tabular}{lcccc}
\hline Term & Count & Genes & P-value & Fold Enrichment \\
\hline Axon guidance & 2 & $\begin{array}{c}E P H A 5 \uparrow \\
S E M A 3 A \uparrow\end{array}$ & $1.2 \mathrm{E}-1$ & 13.6 \\
\hline
\end{tabular}

$\mathrm{EASE}=1.0$

inhibitor in the osteogenic differentiation capability of long-term passaged human bone marrow stromal cells $[52,53]$. Although up-regulated EPHA5 in our NGS database of P8 osteoblasts was observed, the association between EPHA5 and other bone diseases or human aging has not been reported.

SEMA3A, a secreted glycoprotein, is known as one of the axon guidance molecules, participating in the development of central and peripheral nervous systems, and impeding axonal regeneration and repair after injury [54-57]. Recently, the role of $S E M A 3 A$ in bone homeostasis has been explicated, and the balance between sensory input and sympathetic output may modulate bone remodeling [54, 58-60]. SEMA3A acts as an autocrine to regulate neuronal development and osteoblastogenesis, and exerts different regulatory effects in various disease entities $[58,61,62]$. The regulation of $S E M A 3 A$ on bone remodeling was speculated to act through sensory innervation [63]. One study investigated the association between $S E M A 3 A$, traumatic brain injury and osteogenesis and proposed the role of $S E M A 3 A$ in facilitating fracture healing in traumatic brain injury [64]. However, there is not much literature reporting the association between $S E M A 3 A$ sensory innervation of bone and formation
A miRmap

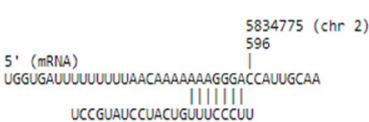

uccGuauccuacugulucccul
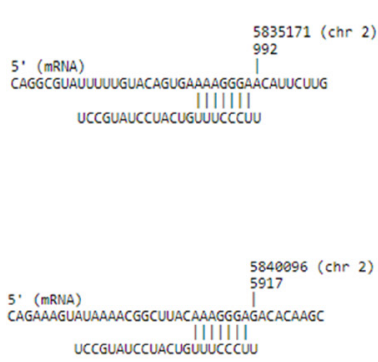

B

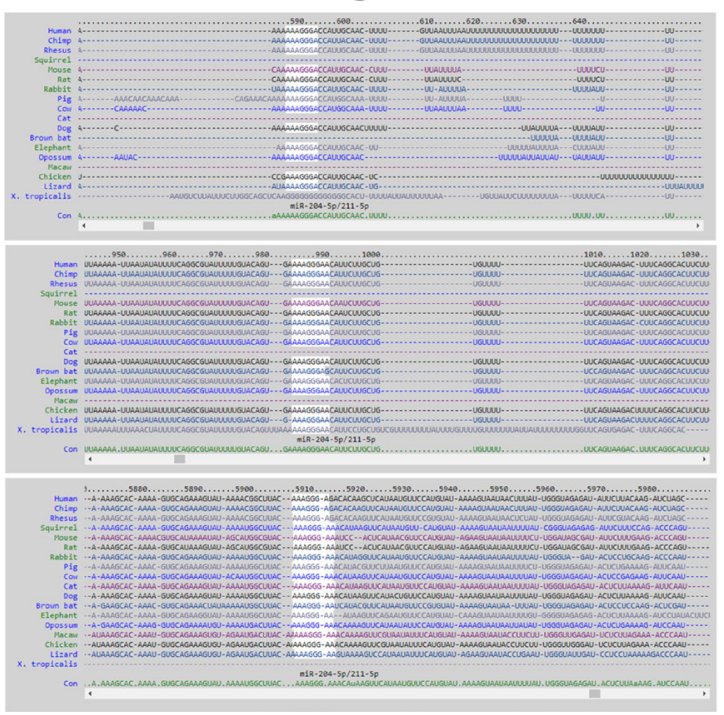

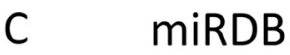

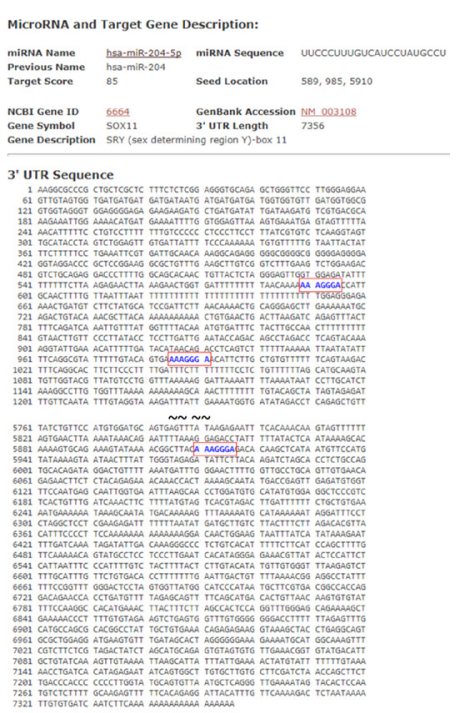

Figure 5: The putative binding site of SOX11 for miR-204-5p. The target binding site and sequence alignment of miR-204-5p on SOX11 3'UTR at positions of 589-595, 985-991 and 5910-5916 were validated in three microRNA target prediction databases, including miRmap (A), TargetScan (B) and $\operatorname{miRDB}(\mathbf{C})$. 
of neurogenic HO. Since early inflammatory signs of HO usually mimic other clinical conditions such as thrombophlebitis and cellulitis, and the maturation of HO formation varies among patients with CNS injury, our current findings provide novel insight in studying the role of $S E M A 3 A$ as a biomarker in the early diagnosis and evaluation of maturation and treatment efficacy of neurogenic $\mathrm{HO}$, and merit further comprehensive investigation.

In conclusion, our study shows that miR-204$5 \mathrm{p}-S O X 11$ regulation plays an important role in the homeostasis of bone in aging osteoblasts, and SEMA3A

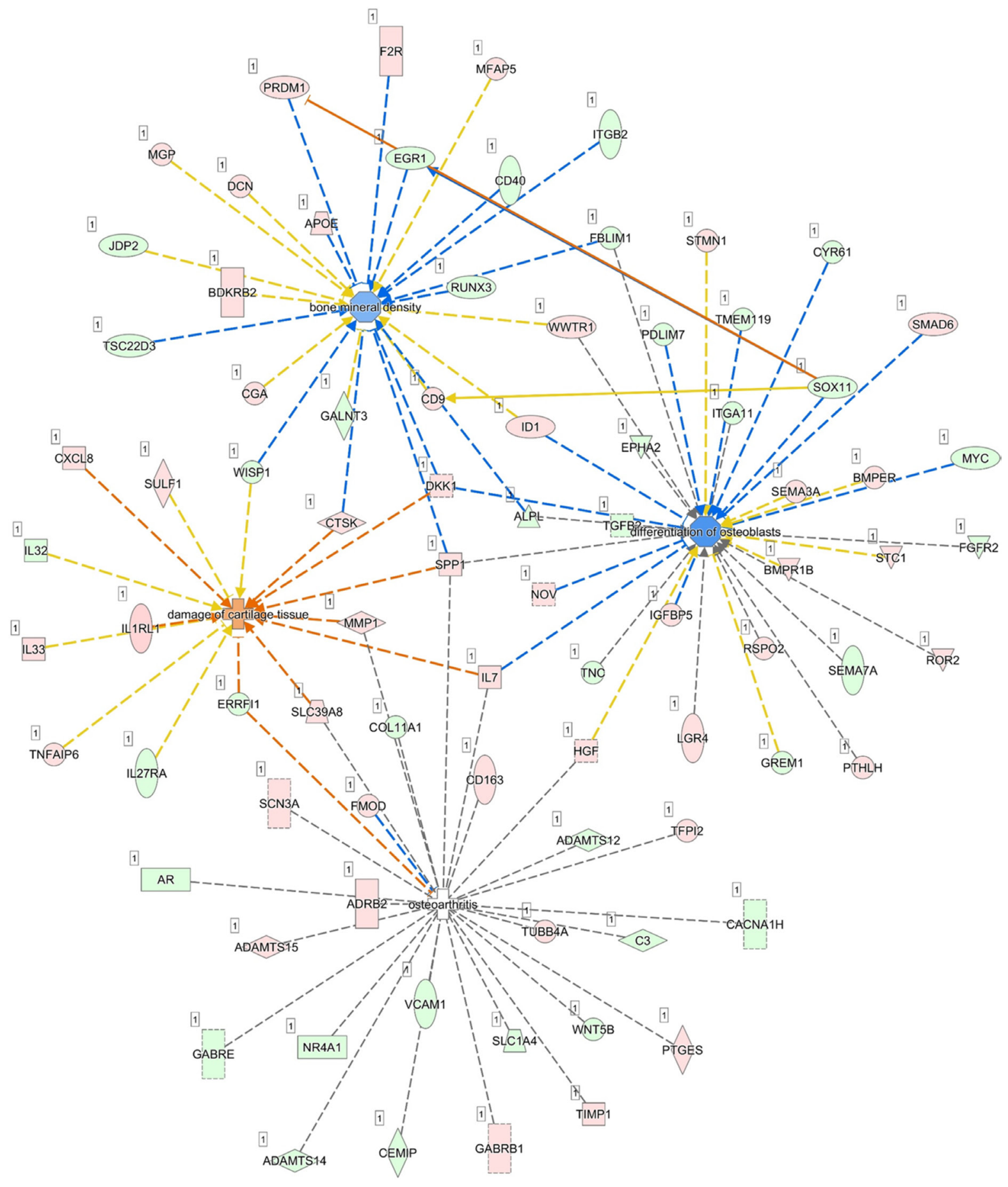

Figure 6: Merged skeletal diseases and functions network and prediction of target molecules. The differentially expressed genes identified in our NGS dataset were analyzed by IPA and categorized into 25 networks, where skeletal diseases and functions annotation, including bone mineral density, differentiation of osteoblasts, osteoarthritis and damage of cartilage tissue were selected to identify related genes. Merged network analysis showed SOX11 was interconnected between the networks of osteoblast differentiation and bone mineral density, and was predicted to inhibit EGRI and activate PRDM1, two molecules in the bone mineral density network. 
may exert a potential linkage to the development of neurogenic HO, as summarized in Figure 9. Our findings suggest new candidate genes in the diagnostic evaluation of geriatric musculoskeletal disorders, and provide novel insights into investigation of potential biomarkers in neurogenic $\mathrm{HO}$.

\section{MATERIALS AND METHODS}

\section{Primary cells and simulation of aging osteoblasts}

Human primary osteoblasts were purchased from Lonza (Walkersville, MD, USA) and cultured in specified osteoblast growth medium (Lonza, Walkersville, MD, USA) according to the manufacturer's recommendation. The first passage of primary osteoblasts, delineated as P1, was defined as cells harvested from directly-thawed vials purchased from Lonza under stable growth conditions. To determine the differential expressions of mRNA and microRNA in aging human osteoblasts in our study, the 8th passage of human primary osteoblasts (P8) were harvested for further RNA extraction, which simulated the aging process.

The characteristics of cellular aging at later passages of human osteoblasts were identified by serial observations of the morphological changes using light microscope
(Zeiss Primo Vert, Jena, Germany). In addition, cells of different passages were stained for $\beta$-galactosidase to confirm the characteristic of osteoblast senescence, using the Senescence $\beta$-Galactosidase Staining Kit (Cell Signaling Technology, Danvers, MA, USA), following the instructions provided by the manufacturer.

\section{RNA sequencing}

The expression profiles of mRNA and microRNA were performed with NGS. Total RNA from harvested cells was extracted by Trizol ${ }^{\circledR}$ Reagent (Invitrogen, USA) according to the manufacturer's instructions. The quality of extracted RNA was analyzed by OD260 detection using an ND-1000 spectrophotometer (Nanodrop Technology, USA), and samples were readied for further RNA preparation and sequencing analysis of both RNA-seq and small RNA-seq by Welgene Biotechnology Company (Welgene, Taipei, Taiwan). The quality of extracted RNA was confirmed by RNA integrity number (RIN) using Agilent Bioanalyzer (Agilent Technology, USA). The criteria for differentially expressed mRNAs were set at a fold change of more than 2.0. The criteria for differentially expressed microRNAs were set at fold change of more than 2 and reads per million (RPM) of more than 10.

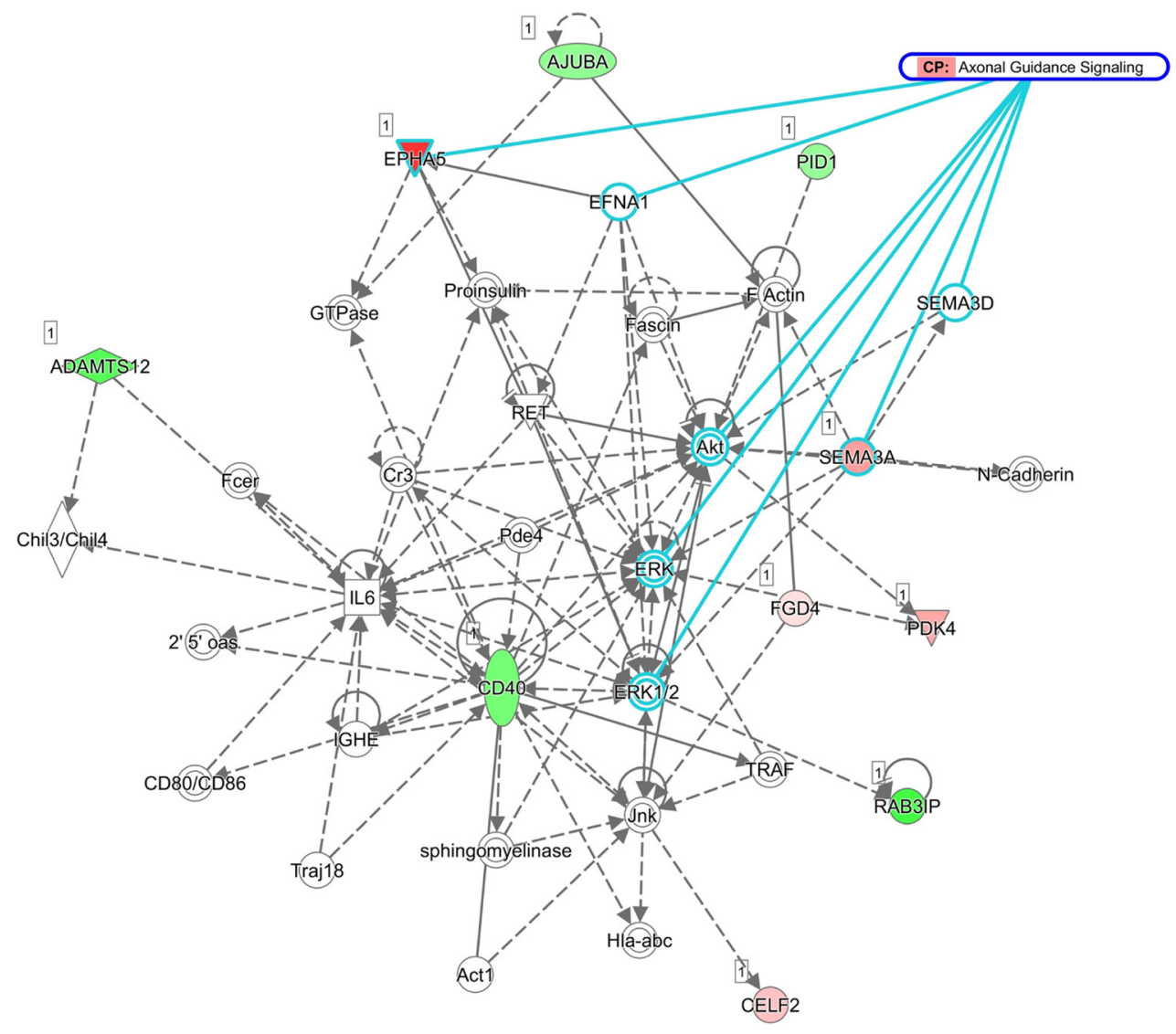

Figure 7: Network of genes potentially involved in axon guidance signaling pathway in aging human osteoblasts. Overlay canonical pathway of axon guidance signaling in network 1 from network analysis of the 22 candidate genes validated the prediction of EPHA5 and SEMA3A involvement in axon guidance pathway from KEGG pathway analysis. 


\section{Ingenuity ${ }^{\circledR}$ Pathway Analysis (IPA)}

Ingenuity ${ }^{\circledR}$ Pathway Analysis (IPA) software (Ingenuity systems, Redwood City, CA, USA) provides a broad spectrum of scientific reports that enable researchers with quick searching. In addition, IPA also provides powerful analysis, integration and interpretation of big data derived from such as RNA-seq, small RNA-seq, and proteomics.

\section{DAVID database}

The DAVID bioinformatics resources provide highthroughput and an integrated data-mining environment to analyze gene lists derived from gene sequencing experiments. Genes of interest uploaded into the DAVID database can be further classified into clusters of different functional annotations, involved pathways and diseases. The Expression Analysis Systematic Explorer (EASE)

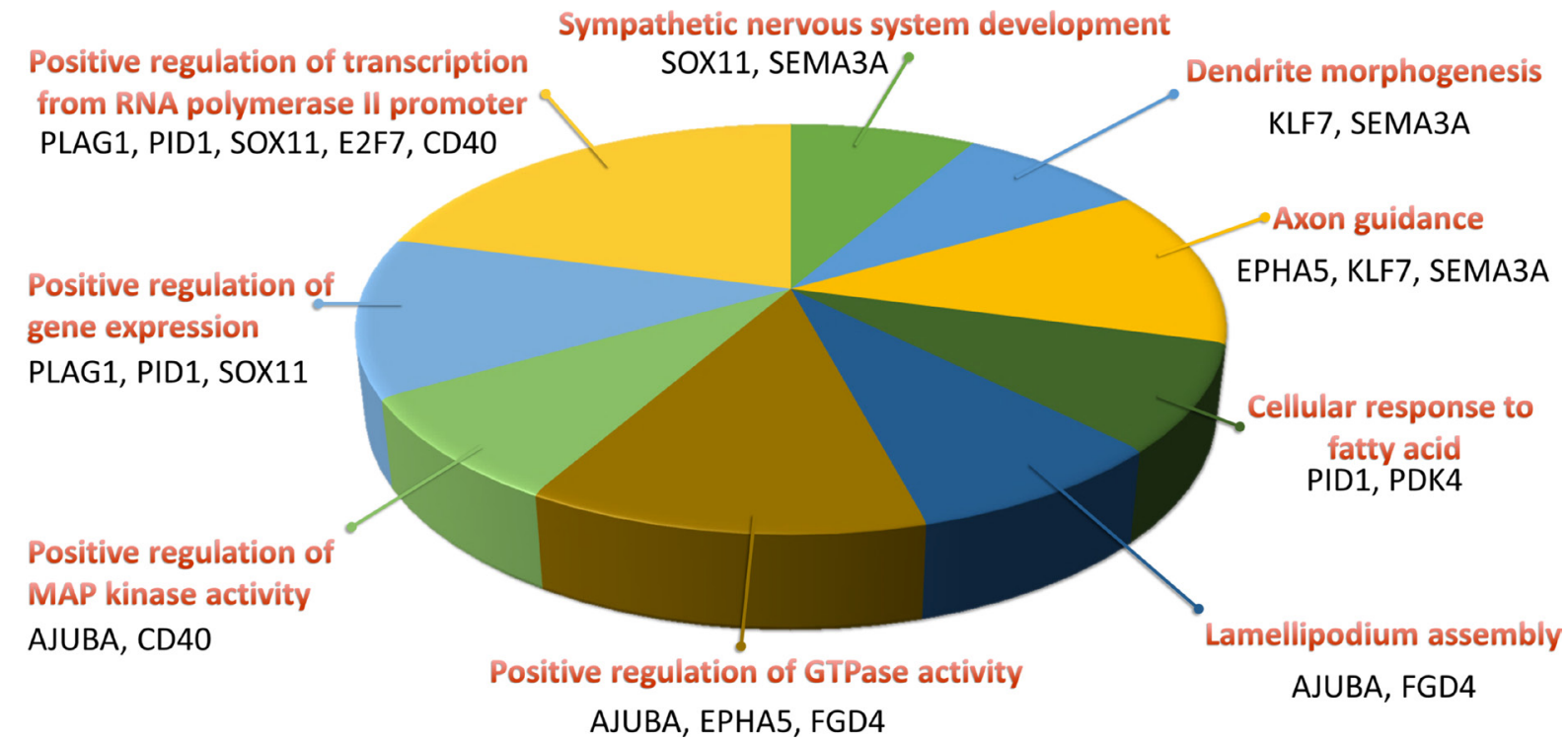

Figure 8: Biological process analysis of candidate genes in aging human osteoblasts. Functional annotation analysis of the 22 candidate genes by the DAVID database identified nine terms of biological processes involved. Four genes (KLF7, SOX11, EPHA5, and $S E M A 3 A$ ) were involved in axon guidance, sympathetic nervous system development and dendrite morphogenesis. The selected criteria for functional annotation analysis were EASE $=0.1$ and fold enrichment $>1.3$.

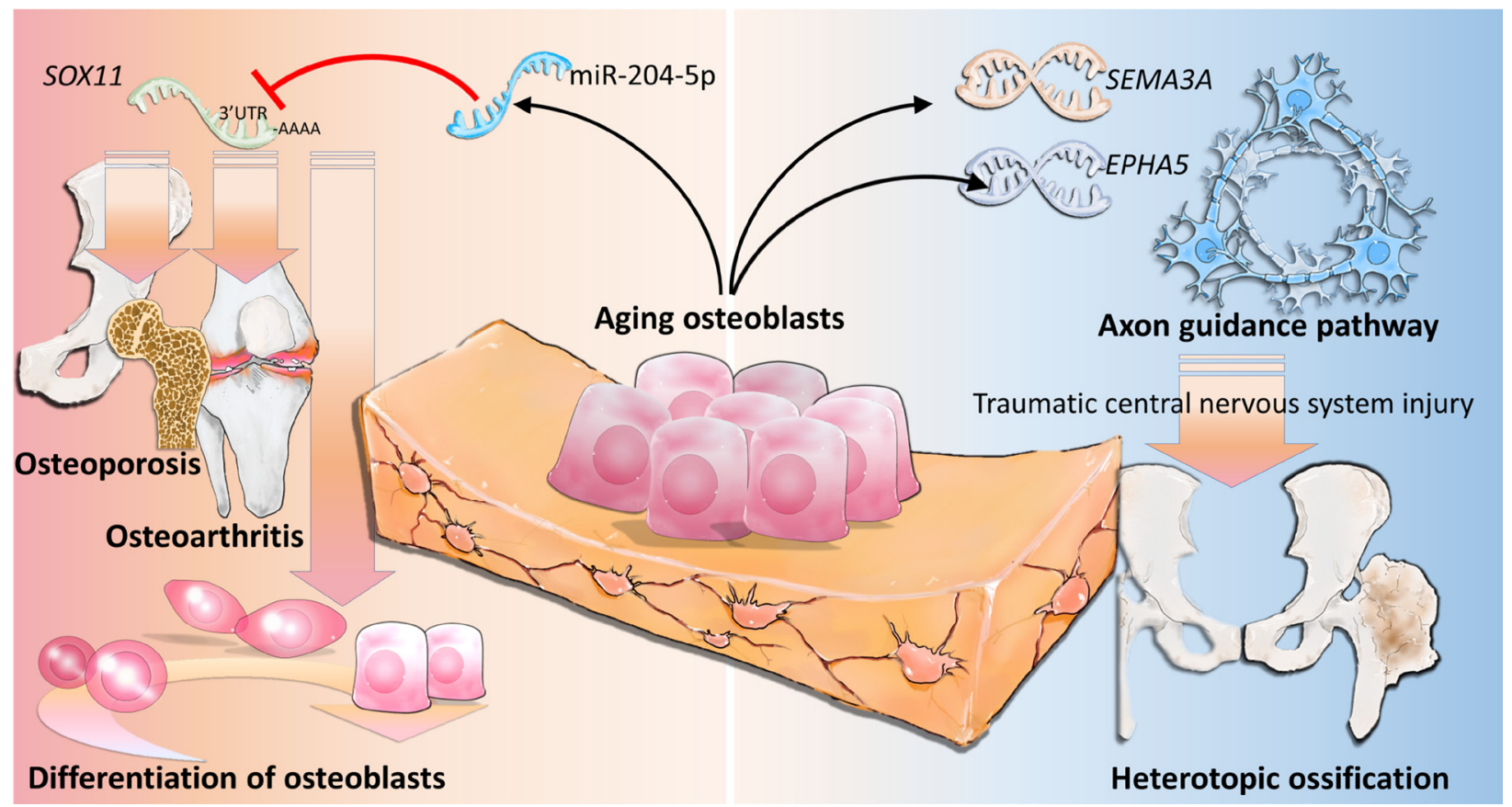

Figure 9: The proposed novel molecular mechanisms of gene regulations involved in aging osteoblasts. 
score represents modified Fisher's exact $p$-value in the DAVID database, and is used to screen for genes potentially involved in specific signaling pathways in our gene list. The default cutoff value of EASE score is set at 0.1 , and adjustable by the users. The higher cutoff value we set, the lower significance of our gene list in a signaling pathway. On the contrary, the lower cutoff value we set, the higher significance of our gene list in a signaling pathway. Considering the complex biological data mining process, the EASE scores suggest, rather than decide, which genes are potentially involved in the pathway, and users are recommended to make further judgment of the biological relevance [19].

\section{Gene Expression Omnibus (GEO)}

GEO is a database that accepts array and sequencebased data. The GEO tool provides researchers specific gene expression profiles in datasets. The array with accession number GSE74209 was used in this study to verify the microRNAs of interest, which consisted of femoral neck trabecular bones from 6 female osteoarthritic patients without osteoporosis, and 6 females with osteoporotic fractures [26].

\section{miRmap database}

MiRmap is an open-source software library, developed by Vejnar et al., that provides higher microRNA target prediction through comprehensive approaches, including thermodynamic, evolutionary, probabilistic and sequence-based approaches. Using comprehensive features with linear model to predict the repression strength of a specific microRNA, input of a microRNA of interest can lead to a list of putative target genes with respective miRmap scores, which indicated the predictive strength of repression. Putative microRNA target genes with miRmap scores higher than 99.0 were selected for this study [21, 65].

\section{Statistical analysis}

The raw data obtained from GEO database were analyzed to compare the expression values of candidate microRNAs between two groups, using the Mann-Whitney $U$ test with IBM SPSS Statistics for Windows, version 19 (IBM Corp., Armonk, NY, USA).

\section{CONFLICTS OF INTEREST}

The authors declare that they have no conflicts of interest.

\section{FUNDING}

This study was supported by grants from the Ministry of Science and Technology (MOST
104-2320-B-037-014-MY3; MOST 104-2314-B-037-053MY4), the Kaohsiung Medical University "Aim for the Top 500 Universities Grant” (Grant No. KMU-TP104D18; KMU-TP105C05), and the "KMU-KMUH Co-Project of Key Research" (Grant No. KMU-DK 107009 from Kaohsiung Medical University).

\section{REFERENCES}

1. Mertz L. The Coming Gray Tide: Wanted: Health Innovations for an Increasingly Older Population. IEEE Pulse. 2017; 8:6-11.

2. Beard JR, Officer A, de Carvalho IA, Sadana R, Pot AM, Michel JP, Lloyd-Sherlock P, Epping-Jordan JE, Peeters G, Mahanani WR, Thiyagarajan JA, Chatterji S. The World report on ageing and health: a policy framework for healthy ageing. Lancet. 2016; 387:2145-54.

3. Khosla S. Pathogenesis of age-related bone loss in humans. J Gerontol A Biol Sci Med Sci. 2013; 68:1226-35.

4. Florencio-Silva R, Sasso GR, Sasso-Cerri E, Simoes MJ, Cerri PS. Biology of Bone Tissue: Structure, Function, and Factors That Influence Bone Cells. Biomed Res Int. 2015; 2015:421746.

5. Sims NA, Gooi JH. Bone remodeling: Multiple cellular interactions required for coupling of bone formation and resorption. Semin Cell Dev Biol. 2008; 19:444-51.

6. Lee WC, Guntur AR, Long F, Rosen CJ. Energy Metabolism of the Osteoblast: Implications for Osteoporosis. Endocr Rev. 2017; 38:255-66.

7. Tran N, Hutvagner G. Biogenesis and the regulation of the maturation of miRNAs. Essays Biochem. 2013; 54:17-28.

8. Sun KT, Chen MY, Tu MG, Wang IK, Chang SS, Li CY. MicroRNA-20a regulates autophagy related proteinATG16L1 in hypoxia-induced osteoclast differentiation. Bone. 2015; 73:145-53.

9. Zhou M, Ma J, Chen S, Chen X, Yu X. MicroRNA-17-92 cluster regulates osteoblast proliferation and differentiation. Endocrine. 2014; 45:302-10.

10. Li XF, Shen WW, Sun YY, Li WX, Sun ZH, Liu YH, Zhang L, Huang C, Meng XM, Li J. MicroRNA-20a negatively regulates expression of NLRP3-inflammasome by targeting TXNIP in adjuvant-induced arthritis fibroblast-like synoviocytes. Joint Bone Spine. 2016; 83:695-700.

11. Iliopoulos D, Malizos KN, Oikonomou P, Tsezou A. Integrative microRNA and proteomic approaches identify novel osteoarthritis genes and their collaborative metabolic and inflammatory networks. PLoS One. 2008; 3:e3740.

12. Gao J, Yang T, Han J, Yan K, Qiu X, Zhou Y, Fan Q, Ma B. MicroRNA expression during osteogenic differentiation of human multipotent mesenchymal stromal cells from bone marrow. J Cell Biochem. 2011; 112:1844-56.

13. Vimalraj S, Selvamurugan N. MicroRNAs expression and their regulatory networks during mesenchymal stem cells differentiation toward osteoblasts. Int J Biol Macromol. 2014; 66:194-202. 
14. Li L, Qi Q, Luo J, Huang S, Ling Z, Gao M, Zhou Z, Stiehler M, Zou X. FOXO1-suppressed miR-424 regulates the proliferation and osteogenic differentiation of MSCs by targeting FGF2 under oxidative stress. Sci Rep. 2017; 7:42331.

15. Kim JH, Lee BR, Choi ES, Lee KM, Choi SK, Cho JH, Jeon WB, Kim E. Reverse Expression of Aging-Associated Molecules through Transfection of miRNAs to Aged Mice. Mol Ther Nucleic Acids. 2017; 6:106-15.

16. Kangas R, Tormakangas T, Fey V, Pursiheimo J, Miinalainen I, Alen M, Kaprio J, Sipila S, Saamanen AM, Kovanen $\mathrm{V}$, Laakkonen EK. Aging and serum exomiR content in women-effects of estrogenic hormone replacement therapy. Sci Rep. 2017; 7:42702.

17. Li T, Yan X, Jiang M, Xiang L. The comparison of microRNA profile of the dermis between the young and elderly. J Dermatol Sci. 2016; 82:75-83.

18. Zhao M, Liu D, Qu H. Systematic review of next-generation sequencing simulators: computational tools, features and perspectives. Brief Funct Genomics. 2017; 16:121-8.

19. Huang da W, Sherman BT, Lempicki RA. Systematic and integrative analysis of large gene lists using DAVID bioinformatics resources. Nat Protoc. 2009; 4:44-57.

20. Clough E, Barrett T. The Gene Expression Omnibus Database. Methods Mol Biol. 2016; 1418:93-110.

21. Vejnar CE, Zdobnov EM. MiRmap: comprehensive prediction of microRNA target repression strength. Nucleic Acids Res. 2012; 40:11673-83.

22. Kassem M, Ankersen L, Eriksen EF, Clark BF, Rattan SI. Demonstration of cellular aging and senescence in serially passaged long-term cultures of human trabecular osteoblasts. Osteoporos Int. 1997; 7:514-24.

23. Kim JY, Park YK, Lee KP, Lee SM, Kang TW, Kim HJ, Dho SH, Kim SY, Kwon KS. Genome-wide profiling of the microRNA-mRNA regulatory network in skeletal muscle with aging. Aging (Albany NY). 2014; 6:524-44. https:// doi.org/10.18632/aging.100677.

24. Portal-Nunez S, Esbrit P, Alcaraz MJ, Largo R. Oxidative stress, autophagy, epigenetic changes and regulation by miRNAs as potential therapeutic targets in osteoarthritis. Biochem Pharmacol. 2016; 108:1-10.

25. Marini F, Cianferotti L, Brandi ML. Epigenetic Mechanisms in Bone Biology and Osteoporosis: Can They Drive Therapeutic Choices? Int J Mol Sci. 2016; 17.

26. De-Ugarte L, Yoskovitz G, Balcells S, Guerri-Fernandez R, Martinez-Diaz S, Mellibovsky L, Urreizti R, Nogues X, Grinberg D, Garcia-Giralt N, Diez-Perez A. MiRNA profiling of whole trabecular bone: identification of osteoporosis-related changes in MiRNAs in human hip bones. BMC Med Genomics. 2015; 8:75.

27. Valenti MT, Dalle Carbonare L, Mottes M. Osteogenic Differentiation in Healthy and Pathological Conditions. Int J Mol Sci. 2016; 18.

28. Kassem M, Marie PJ. Senescence-associated intrinsic mechanisms of osteoblast dysfunctions. Aging Cell. 2011; 10:191-7.
29. Luo YH, Tang W, Zhang X, Tan Z, Guo WL, Zhao N, Pang SM, Dang YW, Rong MH, Cao J. Promising significance of the association of miR-204-5p expression with clinicopathological features of hepatocellular carcinoma. Medicine (Baltimore). 2017; 96:e7545.

30. Luan W, Qian Y, Ni X, Bu X, Xia Y, Wang J, Ruan H, Ma S, $\mathrm{Xu}$ B. miR-204-5p acts as a tumor suppressor by targeting matrix metalloproteinases-9 and B-cell lymphoma-2 in malignant melanoma. Onco Targets Ther. 2017; 10:123746.

31. Liu L, Wang J, Li X, Ma J, Shi C, Zhu H, Xi Q, Zhang J, Zhao X, Gu M. MiR-204-5p suppresses cell proliferation by inhibiting IGFBP5 in papillary thyroid carcinoma. Biochem Biophys Res Commun. 2015; 457:621-6.

32. Huang J, Zhao L, Xing L, Chen D. MicroRNA-204 regulates Runx2 protein expression and mesenchymal progenitor cell differentiation. Stem Cells. 2010; 28:357-64.

33. Semeghini MS, de Azevedo FG, Fernandes RR, Assis AF, Dernowsek JA, Rosa AL, Siessere S, Passos GA, BombonatoPrado KF. Menopause transition promotes distinct modulation of mRNAs and miRNAs expression in calvaria and bone marrow osteoblastic cells. Cell Biol Int. 2017 Jun 2. https:// doi.org/10.1002/cbin.10802. [Epub ahead of print].

34. Bhattaram P, Kato K, Lefebvre V. Progenitor cell fate, SOXC and WNT. Oncotarget. 2015; 6:24596-7. https://doi. org/10.18632/oncotarget.5237.

35. Lefebvre V, Bhattaram P. SOXC Genes and the Control of Skeletogenesis. Curr Osteoporos Rep. 2016; 14:32-8.

36. Sock E, Rettig SD, Enderich J, Bosl MR, Tamm ER, Wegner M. Gene targeting reveals a widespread role for the high-mobility-group transcription factor Sox11 in tissue remodeling. Mol Cell Biol. 2004; 24:6635-44.

37. Gadi J, Jung SH, Lee MJ, Jami A, Ruthala K, Kim KM, Cho NH, Jung HS, Kim CH, Lim SK. The transcription factor protein Sox11 enhances early osteoblast differentiation by facilitating proliferation and the survival of mesenchymal and osteoblast progenitors. J Biol Chem. 2013; 288:25400-13.

38. Kan A, Ikeda T, Fukai A, Nakagawa T, Nakamura K, Chung UI, Kawaguchi H, Tabin CJ. SOX11 contributes to the regulation of GDF5 in joint maintenance. BMC Dev Biol. 2013; 13:4.

39. Xu L, Huang S, Hou Y, Liu Y, Ni M, Meng F, Wang K, Rui Y, Jiang X, Li G. Sox11-modified mesenchymal stem cells (MSCs) accelerate bone fracture healing: Sox11 regulates differentiation and migration of MSCs. Faseb j. 2015; 29:1143-52.

40. Hempel A, Pagnamenta AT, Blyth M, Mansour S, McConnell V, Kou I, Ikegawa S, Tsurusaki Y, Matsumoto N, Lo-Castro A, Plessis G, Albrecht B, Battaglia A, et al. Deletions and de novo mutations of SOX11 are associated with a neurodevelopmental disorder with features of CoffinSiris syndrome. J Med Genet. 2016; 53:152-62.

41. Zhang X, Chen K, Wei B, Liu X, Lei Z, Bai X. Ginsenosides $\mathrm{Rg} 3$ attenuates glucocorticoid-induced osteoporosis through regulating BMP-2/BMPR1A/Runx2 signaling pathway. Chem Biol Interact. 2016; 256:188-97. 
42. Wang X, Manner PA, Horner A, Shum L, Tuan RS, Nuckolls GH. Regulation of MMP-13 expression by RUNX2 and FGF2 in osteoarthritic cartilage. Osteoarthritis Cartilage. 2004; 12:963-73.

43. Donnellan R, Chetty R. Cyclin D1 and human neoplasia. Mol Pathol. 1998; 51:1-7.

44. McCulloch K, Litherland GJ, Rai TS. Cellular senescence in osteoarthritis pathology. Aging Cell. 2017; 16:210-8.

45. He H, Chen K, Wang F, Zhao L, Wan X, Wang L, Mo Z. miR-204-5p promotes the adipogenic differentiation of human adipose-derived mesenchymal stem cells by modulating DVL3 expression and suppressing Wnt/betacatenin signaling. Int J Mol Med. 2015; 35:1587-95.

46. Zhu X, Yang S, Lin W, Wang L, Ying J, Ding Y, Chen X. Roles of Cell Cyle Regulators Cyclin D1, CDK4, and p53 in Knee Osteoarthritis. Genet Test Mol Biomarkers. 2016; 20:529-34.

47. Bargellesi S, Cavasin L, Scarponi F, Tanti A, Bonaiuti D, Bartolo M, Boldrini P, Estraneo A. Occurrence and predictive factors of heterotopic ossification in severe acquired brain injured patients during rehabilitation stay: cross-sectional survey. Clin Rehabil. 2017: 269215517723161 .

48. Vanden Bossche L, Vanderstraeten G. Heterotopic ossification: a review. J Rehabil Med. 2005; 37:129-36.

49. Salisbury E, Rodenberg E, Sonnet C, Hipp J, Gannon FH, Vadakkan TJ, Dickinson ME, Olmsted-Davis EA, Davis AR. Sensory nerve induced inflammation contributes to heterotopic ossification. J Cell Biochem. 2011; 112:2748-58.

50. Lazard ZW, Olmsted-Davis EA, Salisbury EA, Gugala Z, Sonnet C, Davis EL, Beal E 2nd, Ubogu EE, Davis AR. Osteoblasts Have a Neural Origin in Heterotopic Ossification. Clin Orthop Relat Res. 2015; 473:2790-806.

51. Davis EL, Davis AR, Gugala Z, Olmsted-Davis EA. Is het erotopic ossification getting nervous?: The role of the peripheral nervous system in heterotopic ossification. Bone. 2017 Jul 15. https://doi.org/10.1016/j.bone.2017.07.016. [Epub ahead of print].

52. Yamada T, Yuasa M, Masaoka T, Taniyama T, Maehara H, Torigoe I, Yoshii T, Shinomiya K, Okawa A, Sotome S. After repeated division, bone marrow stromal cells express inhibitory factors with osteogenic capabilities, and EphA5 is a primary candidate. Bone. 2013; 57:343-54.

53. Tanabe S, Sato Y, Suzuki T, Suzuki K, Nagao T, Yamaguchi T. Gene expression profiling of human mesenchymal stem cells for identification of novel markers in early- and latestage cell culture. J Biochem. 2008; 144:399-408.
54. Xu R. Semaphorin 3A: A new player in bone remodeling. Cell Adh Migr. 2014; 8:5-10.

55. Hou ST, Nilchi L, Li X, Gangaraju S, Jiang SX, Aylsworth A, Monette R, Slinn J. Semaphorin3A elevates vascular permeability and contributes to cerebral ischemia-induced brain damage. Sci Rep. 2015; 5:7890.

56. Quinta HR, Pasquini JM, Rabinovich GA, Pasquini LA. Glycan-dependent binding of galectin-1 to neuropilin-1 promotes axonal regeneration after spinal cord injury. Cell Death Differ. 2014; 21:941-55.

57. Tannemaat MR, Korecka J, Ehlert EM, Mason MR, van Duinen SG, Boer GJ, Malessy MJ, Verhaagen J. Human neuroma contains increased levels of semaphorin $3 \mathrm{~A}$, which surrounds nerve fibers and reduces neurite extension in vitro. J Neurosci. 2007; 27:14260-4.

58. Li Z, Hao J, Duan X, Wu N, Zhou Z, Yang F, Li J, Zhao Z, Huang S. The Role of Semaphorin 3A in Bone Remodeling. Front Cell Neurosci. 2017; 11:40.

59. Hayashi M, Nakashima $T$, Taniguchi $M$, Kodama $T$, Kumanogoh A, Takayanagi H. Osteoprotection by semaphorin 3A. Nature. 2012; 485:69-74.

60. Li Y, Yang L, He S, Hu J. The effect of semaphorin $3 \mathrm{~A}$ on fracture healing in osteoporotic rats. J Orthop Sci. 2015; 20:1114-21.

61. Takagawa S, Nakamura F, Kumagai K, Nagashima Y, Goshima Y, Saito T. Decreased semaphorin3A expression correlates with disease activity and histological features of rheumatoid arthritis. BMC Musculoskelet Disord. 2013; $14: 40$.

62. Okubo M, Kimura T, Fujita Y, Mochizuki S, Niki Y, Enomoto H, Suda Y, Toyama Y, Okada Y. Semaphorin 3A is expressed in human osteoarthritic cartilage and antagonizes vascular endothelial growth factor 165-promoted chondrocyte migration: an implication for chondrocyte cloning. Arthritis Rheum. 2011; 63:3000-9.

63. Fukuda T, Takeda S, Xu R, Ochi H, Sunamura S, Sato T, Shibata S, Yoshida Y, Gu Z, Kimura A, Ma C, Xu C, Bando $\mathrm{W}$, et al. Sema3A regulates bone-mass accrual through sensory innervations. Nature. 2013; 497:490-3.

64. Zhang L, Zhang L, Mao Z, Tang P. Semaphoring 3A: an association between traumatic brain injury and enhanced osteogenesis. Med Hypotheses. 2013; 81:713-4.

65. Vejnar CE, Blum M, Zdobnov EM. miRmap web: Comprehensive microRNA target prediction online. Nucleic Acids Res. 2013; 41:W165-8. 